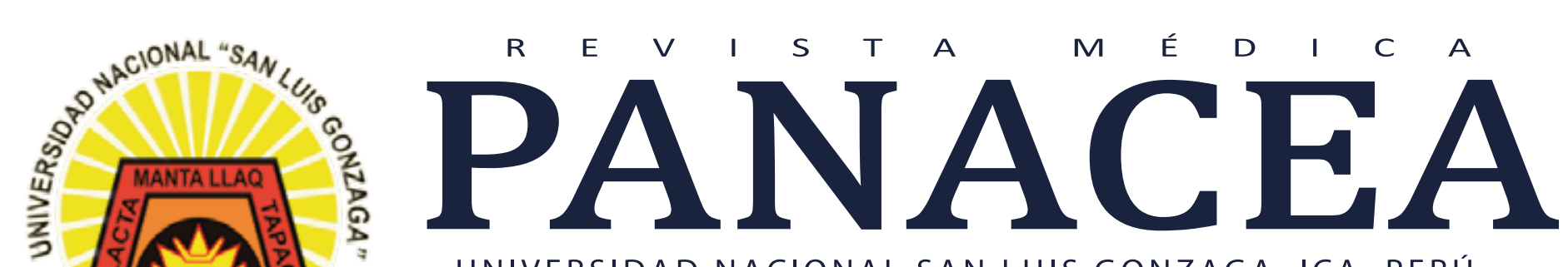

UNIVERSIDAD NACIONAL SAN LUIS GONZAGA. ICA, PERÚ

FACULTAD DE MEDICINA HUMANA "DANIEL ALCIDES CARRIÓN"

p-ISSN 2223-2893

e-ISSN 2225-6989

Volumen 9 Número 3 PUBLICACION CUATRIMESTRAL Setiembre - Diciembre 2020

ARTÍCULO DE REVISIÓN:

\title{
RETARDO DEL CRECIMIENTO FETAL: DIAGNÓSTICO Y MANEJO OBSTÉTRICO.
}

FETAL GROWTH DELAY: DIAGNOSIS AND OBSTETRIC MANAGEMENT.

\section{AUTORES:}

EDITH JULIANA APARCANA PISCONTE

JORGE YBASETA MEDINA

INDEXADA EN:

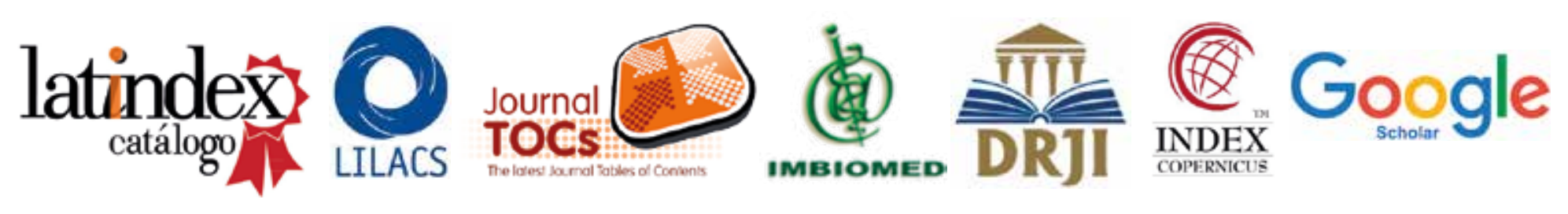

revistas.unica.edu.pe 


\title{
(C) Este trabajo es licenciado Commons Atribución - No Comercial - 4.0 \\ RETARDO DEL CRECIMIENTO FETAL: DIAGNÓSTICO Y MANEJO OBSTÉTRICO.
}

\section{Fetal growth delay: diagnosis and obstetric management.}

\author{
Edith Juliana Aparcana-Pisconte ${ }^{1,2, a, b}$, Jorge Ybaseta-Medina ${ }^{1, a, c}$. \\ 1. Facultad de Medicina Humana, Universidad Nacional San Luis Gonzaga \\ 2. Hospital II Huamanga-EsSalud "Carlos Tuppia Godos" \\ a. Ginecologo-obstétra \\ b. ORCID: https://orcid.org/0000-0002-3783-4610 \\ c. ORCID: https://orcid.org/0000-0003-1224-1357
}

DOI: https://doi.org/10.35563/rmp.v9i3.376

\section{Correspondencia:}

Edith Juliana Aparcana Pisconte

Residencial Ica B-10

Celular 956602001

Correo electrónico:

yuly20_05@hotmail.com

Contribuciones de autoría:

- EAP en la concepción y diseño del artículo; redacción del artículo; revisión crítica del artículo y aprobación de la versión final y JYM en el diseño del artículo; revisión crítica del artículo y aprobación de la versión final.

Conflicto de intereses: no existen conflictos de intereses del autor o autores de orden económico, institucional, laboral o personal.

Financiamiento: Autofinanciado.

\section{Cómo citar:}

Aparcana-Pisconte $\quad E$

Ybaseta-Medina J. Retardo del crecimiento fetal: diagnóstico y manejo obstétrico. Rev méd panacea.2020;9(3) 198-205. DOI:

https://doi.org/10.35

563/rmp.v9i3.376

\section{RESUMEN}

Introducción: : Revisar el tema de retardo de crecimiento fetal para orientar al diagnóstico y manejo. Método: Se realizó un estudio descriptivo de búsqueda de literatura publicada en bases de datos como PubMed/MEDLINE, Science, Scielo, Tesis y libros relacionados al tema. Resultados: Se presentó un consenso de los estudios de diferentes artículos, revisiones e información obtenida sobre el diagnóstico y manejo de la Restricción del crecimiento fetal. Conclusiones: El retardo de crecimiento fetal es una enfermedad que presenta morbimortalidad perinatal su manejo debe basarse en los cambios a nivel del Doppler.

Palabras clave: Retraso del crecimiento Fetal, Ultrasonografía, Efecto Doppler. (fuente: DeCS BIREME).

\section{ABSTRACT}

Introduction: Review the topic of fetal growth retardance to guide diagnosis and management. Method: A descriptive literature search study published in databases such as PubMed/MEDLINE, Science, Scielo, Thesis and books related to the topic was conducted. Results: Consensus was presented on studies of different articles, reviews and information obtained on the diagnosis and management of fetal growth restriction. Conclusions: Fetal growth retardation is a disease that presents perinatal morbidity and mortality, its management must be based on changes at the Doppler level.

Keywords: Fetal Growth Retardation , Ultrasonography, Doppler Effec. (Source: MeSH NLM). 


\section{INTRODUCCIÓN}

"El término restricción del crecimiento fetal (RCF) es utilizado para describir fetos con un peso que es menor al percentil 10 para la edad gestacional" (1). "Pequeño para la edad gestacional (PEG) se define como peso al nacer por debajo del percentil 10 para la edad gestacional. Aunque algunos neonatos PEG son constitucionalmente pequeños, RCF y PEG se utilizan a menudo indistintamente" (2).

La restricción del crecimiento fetal es un determinante importante de la morbilidad y mortalidad perinatal. La detección del RCF es un elemento de reconocimiento clave de la atención prenatal. El examen del paciente enfocando el riesgo clínico más el uso del ultrasonido en mujeres de alto riesgo es el estándar de atención en los Estados Unidos y el Reino Unido, pero se sabe que este enfoque tiene baja sensibilidad (3).

Para evaluar clínicamente el crecimiento fetal, se usan el peso fetal estimándose la progresión de altura sínfisis y fondo uterino, la biometría en serie por ultrasonido y la combinación de biometría y velocimetria Doppler seriado (4).

El retardo del crecimiento fetal está asociado a complicaciones indirectas a corto y largo plazo. Dentro de las complicaciones a corto plazo tenemos: la asfixia intraparto, hipoglicemia, hiperbilirrubinemia, hipotermia, sepsis, coagulopatías y prematuridad. A largo plazo se presentan alteraciones en el tono muscular, coeficientes intelectuales disminuidos en comparación de aquellos productos con peso adecuado $(2,5)$.

El propósito de este artículo es revisar el tema de RCF para orientar al diagnóstico y manejo.

\section{MATERIALES Y MÉTODOS}

Se realizó un estudio descriptivo de búsqueda de literatura publicada en bases de datos como PubMed/MEDLINE, Science, Scielo, tesis y libros relacionados al tema. La estrategia de búsqueda utilizó las palabras claves en español e inglés, de 68 artículos open acces de los últimos 5 años se seleccionaron los que generan contribución importante al trabajo.

\section{DEFINICION}

La RCF se define como fracaso del feto para alcanzar su potencial de crecimiento genéticamente determinado $(4,6)$. La falta de consenso en la terminología, etiología y criterios de diagnóstico para la RCF genera incertidumbre para el manejo óptimo (1)

\section{CLASIFICACIÓN DE LA RESTRICCIÓN DEL CRECIMIENTO INTRAUTERINO}

No existe una definición estándar de oro para RCF, el feto o recién nacido se cuantifica según edad gestacional y los que tienen BPN < al percentil 10 se denominan PEG. Y el PEG se convierte en RCF al utilizar otros criterios que diferencien entre PEG y RCF utilizando ya sea factores de riesgo asociados al embarazo o resultado del Doppler.

Entre las bibliografías revisadas es interesante la guía clínica presentada por Scacchi S. et al (8), donde refiere de aquellos fetos que no alcanzan el p10 y establece clasificación.

\section{Clasificación según comportamiento clínico:}

1- “Pequeño para la edad gestacional: (corresponden al 60 $\%)$ son aquellos con peso fetal estimado (PFE) $y / o$ circunferencia abdominal (CA) entre el p3 y p10 con: Valoración anatómica por ultrasonido normal, Doppler normal y valoración prospectiva con persistencia de crecimiento ecográfico en similares percentiles"(8).

2- Retardo del crecimiento fetal: (corresponden al 25\%) una desviación del propio ritmo de crecimiento determinado según su potencial genético; está causado por insuficiencia placentaria. Se define por:

- PFE y/o CA inferior al p3 como único dato positivo

- PFE y/o CA menor al p10 con Doppler patológico:

- $A U$ > p95 y/o AUt > p95 en menores de 32 semanas

- $\mathrm{AU}>$ p95 o ICP < p5 en mayores de 32 semanas

- PFE y/o CA con descenso marcado en su carril de crecimiento en mayores de 32 semanas (caída de 2 cuartiles en dos ecografías separadas por al menos 2 semanas)

3- "Fetos patológicos: (corresponden al 15\%) son aquellos que se asocian con cromosomopatías, anomalías estructurales o infecciones intrauterinas crónicas" $(7,8)$.

\section{Subclasificacion según momento de aparición y proporciones corporales del RCF}

A. Según edad gestacional al momento de aparición de la RCF

- Retardo del crecimiento fetal precoz: Aparece antes de las 32 semanas de edad gestacional, y generalmente se relaciona con la aparición de preeclampsia, alteraciones a nivel placentario y prematuridad $(6,7,8)$.

- Retardo del crecimiento fetal Tardío: Aparece después de la semana 32 de edad gestacional, también se asocia a insuficiencia placentaria, pero en menor grado que el grupo de restricción del crecimiento Intrauterino de inicio precoz. Su asociación con preeclampsia es baja aproximadamente un $10 \%$, y debido a que se origina generalmente por una insuficiencia placentaria leve el Doppler umbilical tiene una sensibilidad baja menor al $30 \%$ y generalmente es normal $(6,7,8)$.

\section{B. Según las proporciones Corporales Fetales}

- Restricción del Crecimiento Intrauterino simétrico: Es aquel que presenta una disminución de forma proporcionada de sus segmentos corporales. Su instalación comienza desde el inicio de la gestación, por lo que el producto tiene una menor cantidad de células. Su etiología se debe principalmente a anormalidades cromosómicas, genéticas o infecciones congénitas $(5,9)$.

- Restricción del Crecimiento Intrauterino Asimétrico: Este tipo se caracteriza porque existe una desproporción en los segmentos corporales del producto; se instaura durante el tercer trimestre, a causa de disminución de nutrientes fetales. En estos fetos el perímetro abdominal es menor, comparado con los fetos de la misma edad gestacional, pero las otras medidas corporales son similares. Se encuentra una cantidad celular normal, pero de menor tamaño $(7,8,9)$. 
- Restricción del Crecimiento Intrauterino mixto: En este tipo se encuentran una disminución de células y también en tamaño, y se da cuando hay una restricción del crecimiento Intrauterino de inicio temprano asociado a una insuficiencia placentaria $(7,8,9)$.

\section{ETIOLOGÍA}

Entre las etiologías de retardo de crecimiento intrauterino se citan causas maternas, fetales y placentarias.

\section{A. Factores Maternos:}

- "Patología hipertensiva: Se presentan en la mayoría de los embarazos que se complican con restricción del crecimiento fetal. Corresponden a patologías relacionadas con una disminución del flujo feto placentario con bajo flujo de oxígeno. Se ha encontrado que hasta 30 a $40 \%$ de los productos con restricción del crecimiento intrauterino presentaban alguna patología hipertensiva" (10)

- "Estilo de vida: Se ha encontrado relación entre el consumo del tabaco, alcohol y cocaína con el retardo de crecimiento intrauterino". $(10,11)$

- "Trastornos autoinmunes: Como el síndrome de anticuerpos antifosfolípidos y el lupus eritematoso sistémico".

- "Trombofilias: La más estudiada ha sido el relacionado con el Factor $V$ de los factores de coagulación. Un metaanálisis publicado en el 2005 por Howley y cols (15), revisó 10 estudios tipo casos y controles y encontró relación entre la presencia de factor $\vee$ de Leiden y restricción del crecimiento feta". (10).

- "Fármacos: Se encontró relación con restricción del crecimiento de los productos cuyas madres consumen medicamentos antineoplásicos, anticonvulsivantes (fenitoína), beta bloqueadores y esteroides (corticoides)" (10)

- "Desnutrición: Dependiendo de la severidad de la desnutrición en la madre y del trimestre de gestación en que se produzca será el impacto sobre el crecimiento fetal" $(10,11)$.

\section{B. Factores Fetales:}

cromosomopatías, genopatías, malformaciones congénitas, embarazos múltiples, infecciones, anemia, etc. $(10,11,12)$

\section{Factores Placentarios:}

"Se involucran a factores que producen insuficiencia placentaria tenemos: Cómo infartos placentarios, vasculitis, placenta previa, inserción anormal del cordón umbilical, anormalidades placentarias en la gestación múltiple y placentas con inserción de arteria umbilical única" $(9,13)$.

"Los trastornos placentarios asociados al Retardo de crecimiento cursan con alteraciones y aumentando su pulsatilidad mediante ecografía Doppler, a nivel de las arterías uterinas. Si este estado de insuficiencia circulatoria feto-placentaria es permanente, el feto trata de adaptarse al disminuir su tasa de crecimiento. A medida que se hace crónica la causa, los mecanismos de adaptación del feto hacen que se disminuya el crecimiento somático, el tamaño del hígado y de los depósitos de grasa" $(9,13,14,15,16)$.

\section{VELOCIMETRIA DOPPLER EN EL RCF}

Cuando la placenta invade la decidua del miometrio uterino, las arterias sufren grandes cambios haciéndolas semejantes a vasos venosos. Estos cambios se definen al finalizar el primer trimestre de la gestación, haciéndose posible su seguimiento mediante ecografía con Doppler.

Arterias uterinas (Figura 1):

La evaluación Doppler de las arterias uterinas en el primer trimestre es un método indirecto para examinar la circulación uteroplacentaria y puede ser considerado como una herramienta importante en el tamizaje para el desarrollo de preeclampsia y restricción del crecimiento intrauterino para poder ser utilizado en la clínica $(4,16)$

A medida que avanza el embarazo la disminución de la resistencia vascular se refleja en el incremento del flujo en diástole. Una onda anormal se caracteriza por altos índices de pulsatilidad y puede encontrarse alterada en presencia de una arteria umbilical normal. La arteria uterina adquiere especial importancia en la valoración del riesgo del RCF tardío $(10,16)$, se debe utilizar el promedio del IP de ambas arterias uterinas, independientemente de si presentan notch. Se ha visto cómo la presencia de las arteria uterinas con un IP > p95 al momento del diagnóstico tiene una sensibilidad para identificar resultados adversos de $37,7 \%$ con especificidad de $70 \%$, valor predictivo positivo de 91,8 y valor predictivo negativo de $11,1 \%(16)$

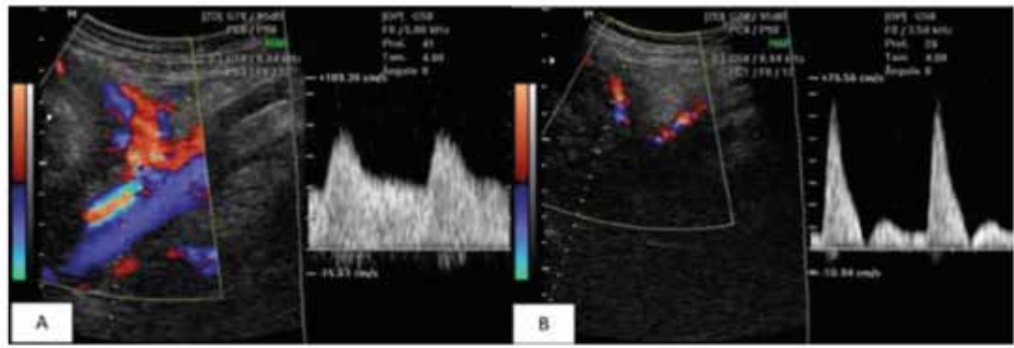

Figura 1. Flujo de Arteria Uterina normal y B. Flujo de Arteria Uterina Patológica. Tomado de Pimiento L. y Beltrán M. Restricción del crecimiento Intrauterino: Una aproximación al Diagnóstico, seguimiento y manejo. Rev. Chilena Obstétrica Ginecológica 2015; 80(6): 493-502.(10)

\section{Arteria umbilical (Figura 2):}

"Uno de los primeros vasos en el estudio de la restricción del crecimiento fetal es la arteria umbilical, al aportar importante información sobre esta patología. En ella se valora el flujo de la circulación (que es de baja impedancia), donde conforme avanza la edad gestacional, se incrementa el flujo diastólico. Los cambios que se dan en este vaso se deben muchas veces por varias enfermedades, que producen cambios en la musculatura lisa de las arteriolas de las vellosidades placentarias" $(16,17)$. 
"La resistencia al flujo sanguíneo que pasa de la arteria umbilical a la placenta puede ser identificado mediante Doppler en forma de reducción o ausencia del flujo diastólico, y en casos muy severos se puede evidenciar hasta reversión del flujo diastólico.

La ausencia y el flujo diastólico reverso en la arteria umbilical indican un estado avanzado de compromiso placentario, se asocian con un mal estado del producto y es generalmente consecuencia a una enfermedad placentaria" $(16,17)$.

"La monitorización prenatal con Doppler de la arteria umbilical ha disminuido los resultados perinatales adversos en embarazos con RCF. El Colegio Americano de Obstetras y Ginecólogos y la Sociedad de Medicina Materno-Fetal han publicado pautas que abogan por el uso de la relación sistólica / diastólica de la arteria umbilical para determinar el momento del parto de estos embarazos. Estas recomendaciones incluyen el parto a las 37 semanas de gestación si las proporciones sistólicas / diastólica son elevadas (percentil 95 para la edad gestacional o más) y entre las 38 y 39 semanas de gestación si las proporciones sistólicas / diastólica son normales (menos del percentil 95 para la edad gestacional)" $(16,17)$.

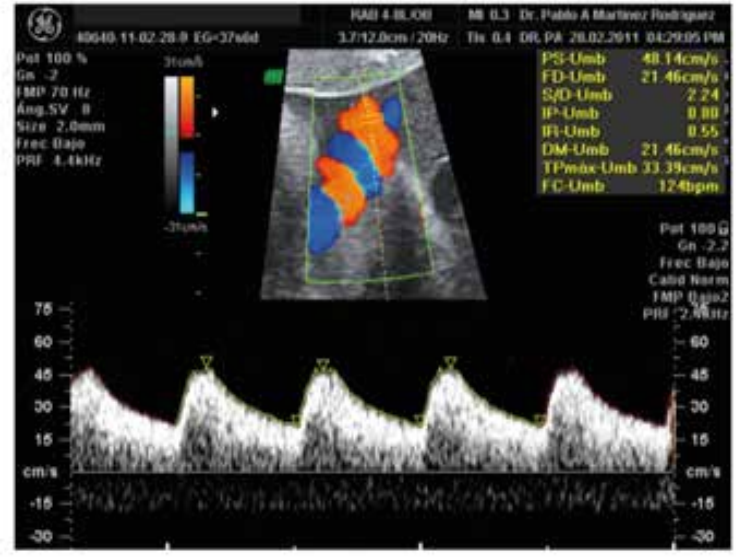

Figura 2. Arteria Umbilical Normal. Tomado de: Martínez P. y Oliva L. Flujometría Doppler en Medicina Materno Fetal. REV MED HONDUR. 2014;82(1):28-32. (18)

\section{E. La arteria cerebral media:}

"La evaluación del Doppler de la arteria cerebral media (MCA) también proporciona información sobre el estado hemodinámico del feto. El cerebro fetal en embarazos sin complicaciones tiene una circulación de alta resistencia. Con la hipoxia progresiva, el flujo sanguíneo aumenta para compensar la disminución del oxígeno disponible (efecto conservador del cerebro). Esto da como resultado una reducción en los parámetros Doppler utilizados para evaluar el flujo sanguíneo a través de la MCA: el pico sistólico a fin diastólico índice de velocidad del flujo sanguíneo (S / D), índice de resistencia e índice de pulsatilidad. Posterior normalización de los índices puede ocurrir cuando la respuesta autorreguladora se vuelve disfuncional"(19).

"No hay evidencia convincente de que el examen del Doppler MCA solo sea útil para orientar las decisiones clínicas sobre el momento oportuno del parto, aunque las alteraciones del Doppler de la MCA pueden ser útiles como complemento del examen Doppler de la arteria umbilical para evaluar la gravedad de la hipoxia y predecir el resultado neonatal" (20).

\section{F. Ductus venoso (Figura 3):}

A la evaluación del Doppler del ductus venoso proporciona información sobre el estado hemodinámico del feto, pero no hay evidencia convincente de que esta información sea útil para orientar las decisiones clínicas sobre el momento del parto (21).

"El flujo sanguíneo del DV se sabe que incrementa durante la hipoxemia; mientras que el flujo a través del hígado fetal disminuye. El mecanismo de control del DV es parcialmente conocido y se ha descrito como un esfínter anatómico que puede afectarse por factores neuronales u hormonales" (21).

"La apertura del DV en hipoxia es un signo de respuesta fetal al estrés. El vaso debe también dilatarse ante el incremento de la presión venosa central. Se ha encontrado que, durante la hipoxemia el flujo sanguíneo de sangre oxigenada se incrementa a través del foramen oval y por lo tanto en la parte superior del cuerpo fetal" (21).

"El DV juega entonces un papel importante en el mantenimiento del oxígeno para órganos fetales vitales, como el cerebro y el corazón. El feto reacciona centralizando la circulación, dando un incremento del flujo sanguíneo a órganos vitales. La hipoxemia permite incrementar la presión umbilical venosa con un incremento relativo en el flujo sanguíneo a través del DV y disminuir el flujo sanguíneo hepático. La restricción en la perfusión hepática hace que se incremente la resistencia en el hígado pudiendo entonces permitir el incremento en el flujo del DV y ayudar a mantener estable el flujo a órganos vitales" (22).

"Aunque el uso del Doppler venoso sigue siendo en gran medida en fase de investigación, un número cada vez mayor de especialistas en medicina materno-fetal está utilizando esta herramienta para evitar un parto muy prematuro en fetos con diastólica final ausente o invertida. El flujo arterial en la arteria umbilical y pruebas fetales antes del parto tranquilizadoras (prueba sin estrés, PBF) y la ausencia de patrones de flujo anormales en el ductus venoso se ha utilizado para respaldar la decisión de extender el embarazo a 32 a 34 semanas" (23).
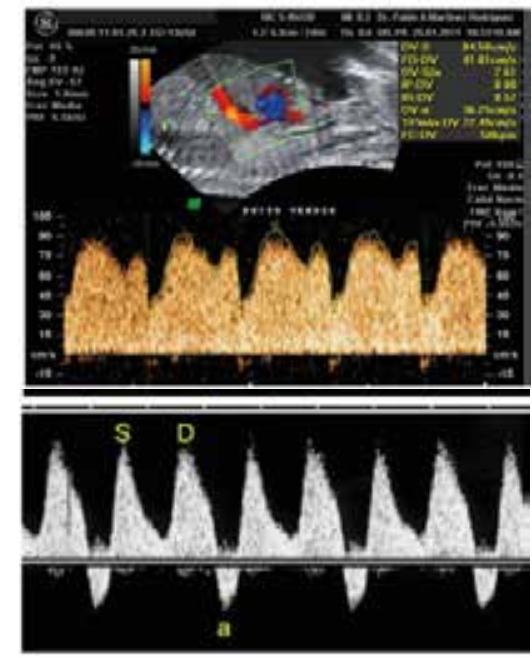

Figura 3. Doppler de Ductus venoso normal (A) Y Ductus Venoso Patológico (B) tomado de Martínez P. y Oliva L. Flujometría Doppler en Medicina Materno Fetal. REV MED HONDUR. 2014;82(1):28-32. 


\section{G. Itsmo aórtico:}

"Este vaso permite conocer el balance entre el flujo del cerebro y el sistema vascular. Cuando hay un aumento de la resistencia a nivel placentario disminuye el flujo diastólico, y en casos más graves aparece un flujo reverso en la diástole siendo un signo de deterioro fetal grave (22).

En los casos de restricción del crecimiento intrauterino a causa de insuficiencia placentaria las alteraciones en el flujo vascular provocan cambios en el istmo aórtico. La resistencia placentaria, que generalmente es la más baja en toda la circulación fetal aumenta, ocasionando que el flujo anterógrado diastólico disminuya, o en casos más graves se produzca un flujo retrogrado a través del istmo aórtico" (24).

"Cuando aparece un flujo reverso a nivel del istmo aórtico, la sangre que proviene de la arteria pulmonar y de la aorta torácica descendente se dirige hacia la placenta, por lo que llega menos cantidad de flujo sanguíneo al cerebro. Debido a que el índice ístmico aórtico es un indicador de cantidad y dirección del flujo, puede ser usado como un marcador clínico en productos con Retardo del Crecimiento Fetal que necesitan ser extraídos del vientre materno antes de que se presente signos de daño cerebral, contra mayor flujo reverso aparece el riesgo de presentar daño cerebral aumenta" $(24,25)$.

Es por esto por lo que la monitorización del patrón de flujo a nivel del Istmo aórtico puede jugar un papel importante en la prevención de secuelas neurológicas posnatales ocasionadas por la lesión hipóxica, incluso, puede brindar datos de forma más temprana que algunos marcadores agudos como el ductus venoso (cuando hay alteraciones a este nivel, ya hay acidemia y necrosis miocárdica) $(24,25)$.

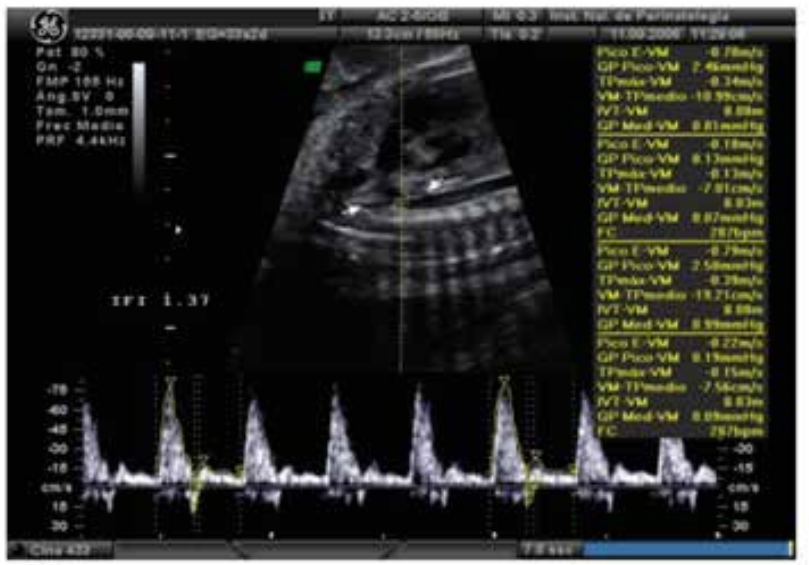

Figura 4. Flujo reverso en Flujometría de Istmo Aórtico en Martínez P. y Oliva L. Flujometría Doppler en Medicina Materno Fetal. REV MED HONDUR. 2014;82(1):28-32.

\section{Índice cerebroplacentario}

"El índice Doppler cerebroplacentario (RCP) es el índice de pulsatilidad (o índice de resistencia) de la MCA dividido por el índice de pulsatilidad de la arteria umbilical (o índice de resistencia). Una RCP baja indica una redistribución del flujo sanguíneo fetal (preservación del cerebro) y es predictivo de resultados neonatales adversos". (26) la tasa de resultados neonatales adversos graves estan mas relacionados cuando el
CPR es mayor a 1. (27) Dentro de los resultados adversos se menciona a: la hemorragia intraventricular, leucomalacia periventricular, encefalopatía isquémica hipóxica, necrosante enterocolitis, displasia broncopulmonar, sepsis y muerte".

La RCP fue más útil para predecir un resultado neonatal adverso cuando el índice de pulsatilidad Doppler de la arteria umbilical es $>95$ percentil (27). El hallazgo adicional de una RCP anormal en estos casos mejora la predicción de un resultado neonatal adverso a un nivel similar al encontrado con flujo diastólico final de la arteria umbilical ausente o invertido.

En otros estudios, una RCP anormal es asociado a una velocidad de crecimiento fetal anormal (27), y tienen mayor riesgo de ingreso en la unidad de cuidados intensivos y parto por cesárea urgente por sufrimiento fetal después de 37 semanas, independientemente del peso fetal (28).

"La RCP puede ser útil para predecir con mayor precisión el riesgo de un resultado adverso en la RCF cuando se usa como complemento del cordón umbilical. el papel potencial de la RCP en el manejo de embarazos complicados por RCF al final del embarazo es importante, pero se requieren estudios adicionales antes de que esta proporción pueda recomendarse para uso clínico de rutina en embarazos con RCF"(28).

\section{Manejo del RCF}

Para un mejor manejo de esta patología, se toma en cuenta la clasificación en estadios según los cambios que se dan en la circulación fetal evaluados mediante Doppler.

\section{Protocolo basado en etapas para controlar el retardo del crecimiento fetal}

"El RCF puede estar entre los problemas obstétricos con mayor variación en la práctica clínica. Esto es el resultado de una combinación de la falta de pruebas sólidas de apoyo, la complejidad de las variables e índices que deben integrarse para evaluar el deterioro fetal y los riesgos variables asociados con la prematuridad en diferentes edades gestacionales"

"Aunque cuando se consideran grupos existen claras diferencias entre las formas de inicio temprano y tardío, de forma individual existe una superposición importante de las características clínicas en las edades gestacionales límite. Además, los casos con la misma edad gestacional al inicio a menudo se detectan en diferentes momentos durante la gestación". (Figueras F. · Gratacós E. 2014). 


\section{ESTADÍOS DE LA EVALUACIÓN DOPPLER EN FETOS CON RCIU}

1. Doppler Tipo 1

- Peso Fetal Estimado < P 3

- Relación Cerebro/Placenta < P5

- IP arteria umbilical > P 95

- IP de ACM < P5

- IP de arterias Uterinas > P 95

2. Doppler Tipo 2

- Peso Fetal Estimado < P 10 asociado a alguno de los siguientes:

- Ausencia de Flujo diastólico en arteria umbilical (>50\% de ciclos en asa libre en ambas arterias, en dos ocasiones $>12$ horas).

- Flujo diastólico reverso en istmo aórtico.
3. Doppler Tipo 3

- Peso Fetal Estimado < P 10 asociado a alguno de los siguientes:

- Flujo diastólico reverso en arteria umbilical (>50\% de ciclos en asa libre en ambas arterias, en dos ocasiones $>$ 12 horas).

- IP de ductus venoso > P 95

4. Doppler tipo 4

- Peso Fetal Estimado < P 10 asociado a alguno de los siguientes:

- Registro cardiotocográfico anormal o patológico (variabilidad < 5 latidos/ minuto y/o patrón de desaceleraciones).

- Flujo diastólico reverso en ductus venoso.

Nota: (P) Percentil. (IP)- Índice de pulsatilidad. (ACM) Arteria cerebral media.

ALGORITMO DE DECISIÓN BASADO EN ETAPAS PARA EL MANEJO DE RCF

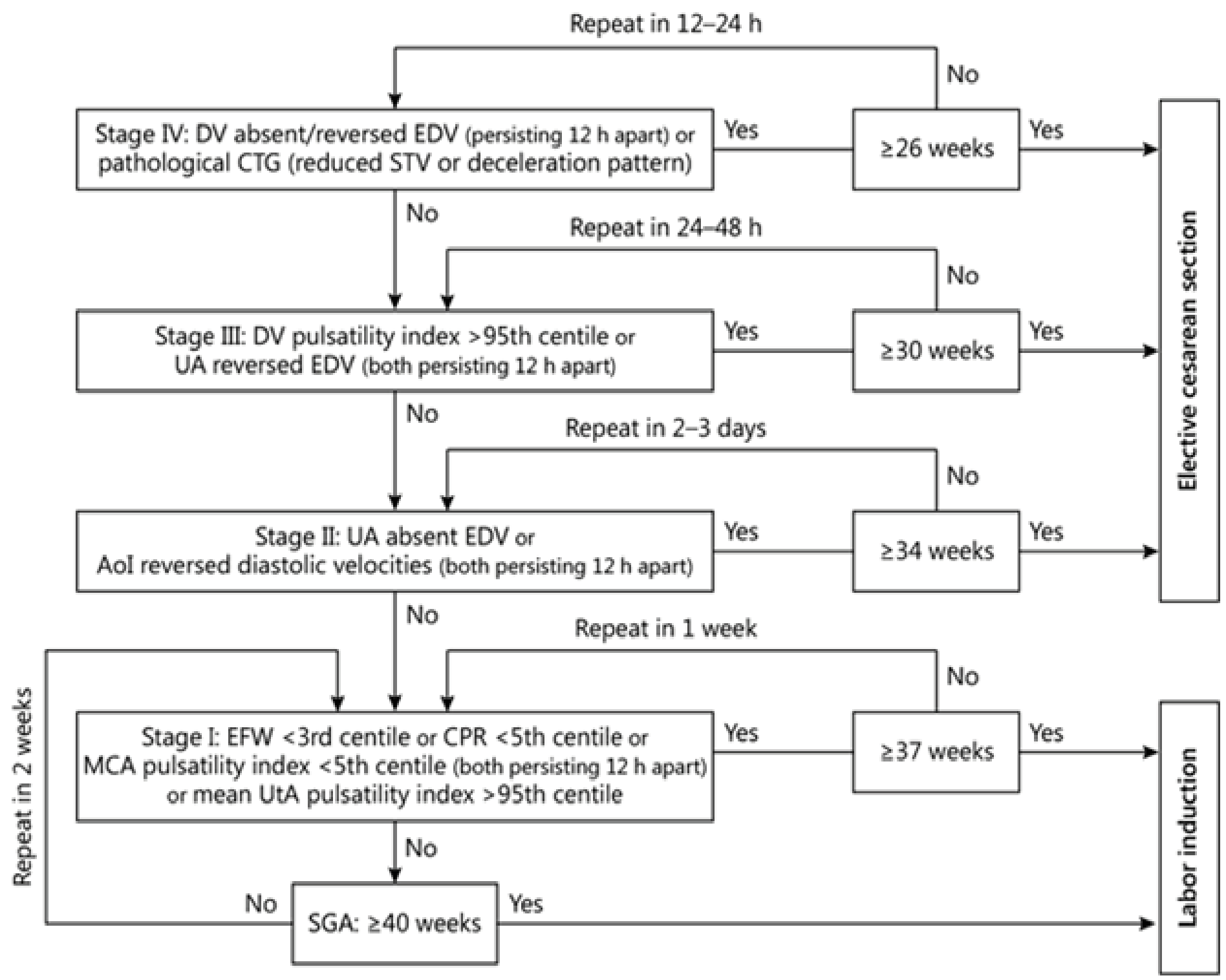

Fuente: Figueras F. · Gratacós E. 2014 (4) 


\section{CONDUCTA EN FETOS CON RCIU PRECOZ}

Una nueva clasificación que permite estandarizar el seguimiento y la conducta en fetos con RCF es la basada en los cambios a nivel del Doppler.

Tabla1: Scacchi M. et al. Hospital Materno Infantil Ramón Sardá

\begin{tabular}{|c|c|c|c|c|c|}
\hline \multicolumn{6}{|c|}{ Recomendaciones para finalización electiva del FRC } \\
\hline Estadío & $\begin{array}{c}\text { Correlato } \\
\text { fisiopatológico }\end{array}$ & Criterios & Seguimiento & $\begin{array}{c}\text { Edad } \\
\text { gestacional }\end{array}$ & Via del parto \\
\hline I-A & Restricción severa & PEF o PA $P<3$ & Semanal & & \\
\hline $\mathrm{I}-\mathrm{B}$ & $\begin{array}{l}\text { del crecimiento } \\
\text { fetal o disfunción } \\
\text { placentaria leve }\end{array}$ & $\begin{array}{c}I C P<5 \\
\text { IP AU P }<95 \\
\text { IP ACM } P<5 \\
\text { IP Aut } P<95\end{array}$ & Cada 72 horas & 37 & Parto \\
\hline II & $\begin{array}{l}\text { Insuficiencia } \\
\text { placentaria }\end{array}$ & $\begin{array}{c}\text { AU con ausencia de flujo } \\
\text { diastólico en mas de los } \\
50 \% \text { de los cortes. }\end{array}$ & Cada 72 horas & 34 & Cesárea \\
\hline III & $\begin{array}{l}\text { Baja sospecha de } \\
\text { acidosis }\end{array}$ & $\begin{array}{l}\text { AU con ausencia de flujo } \\
\text { diastólico reverso } \\
\text { DV con IP P < } 95 \text { o flujo } \\
\text { diastólico ausente }\end{array}$ & Cada 24 horas & 30 & Cesárea \\
\hline II & $\begin{array}{c}\text { Alta sospecha de } \\
\text { acidosis }\end{array}$ & $\begin{array}{l}\text { DV con flujo reverso } \\
\text { NST con perdida de la } \\
\text { variabilidad y } \\
\text { desaceleciones }\end{array}$ & Cada 12 a 24 horas & 26 & Cesárea \\
\hline
\end{tabular}

RCIU precoz y neuroprofilaxis con SO4Mg: "Hay evidencia para recomendar la administración en fetos entre semana 24 y 34 semanas con riesgo inminente de parto, se ha visto una importante disminución de la incidencia de parálisis cerebral en el grupo de fetos tratados, con un mínimo de impregnación de 4 horas antes del parto mejora los resultados"(29).

Recomendación sobre el uso de corticoides para maduración pulmonar: "Se aconseja utilizar en CIR 3 entre 24 y 34 semanas con riesgo elevado de parto en las próximas 48 hs. y en todos los CIR 4 al momento del diagnóstico".

"Se debe tener en cuenta que con la administración de corticoides la mayoría de los fetos con un flujo de fin de diástole ausente en la arteria umbilical presentan una recuperación transitoria de unos 3 - 5 días del flujo diastólico, que es un efecto transitorio y no debe hacer cambiar la conducta" $(8,29)$.

\section{CONDUCTA EN RCIU TARDÍA}

Se divide a los fetos con un peso por debajo del percentilo 10 después de las 34 semanas en dos grupos:

1. PEG: AUt normal, ICP normal y PFE menor al P10 y >P3, el control debe ser cada 2 semanas.
2. RCF: AUt > P95, ICP < P5 y PFE menor al P3, el control debe ser cada semana (Para incluir en el grupo de RCIU se debe dar al menos una de las tres condiciones consignadas) (29)

\section{Recomendaciones:}

a. IP ACM < p5 (signo de vasodilatación cerebral el control es cada 3 o 4 días se debe agregar la evaluación del DV que permitirá predecir la aparición de acidosis.

b. La finalización del embarazo en los fetos PEG se debe realizas mediante la evolución espontanea 40 -41 semanas. c. ICP alterado o arterias uterinas alteradas: inducción con monitoreo a las $37-38$ semanas.

d. ACM con signos de vasodilatación: la finalización se realizará entre las 34-37 semanas mediante inducción con monitoreo, pero se debe tener en cuenta que en estos casos hasta en el $60 \%$ se va a terminar realizando una cesárea de urgencia por pérdida del bienestar fetal. $(4,8,29)$

\section{CONCLUSIONES}

El manejo del RCF precoz teniendo en cuenta los estadios de evaluación brinda mayor facilidad para la toma de decisiones sobre todo al momento de resolver el término de la gestación. Del manejo acertado dependerá efectos adversos en el recién nacido. 


\section{REFERENCIAS BIBLIOGRÁFICAS}

1. American College of Obstetricians and Gynecologists' Committee on Practice Bulletins-Obstetrics and the Society forMaternal-FetalMedicin. ACOG Practice Bulletin No. 204: Fetal Growth Restriction. Obstet Gynecol. 2019;133(2): e97-e109. doi: 10.1097/AOG.0000000000003070.

2. Zhang-Rutledge $K$, Mack LM, Mastrobattista JM, Gandhi M. Significance and Outcomes of Fetal Growth Restriction Below the 5th Percentile Compared to the 5th to 10th Percentiles on Midgestation Growth Ultrasonography. J Ultrasound Med. 2018;37(9):2243-2249. doi: 10.1002/jum.14577. Epub 2018 Feb 24.

3. Gaccioli, Francesca, Irving L.M.H, Sovio Ulla, Charnock-Jones S, Gordon C.S. Screening for fetal growth restriction using fetal biometry combined with maternal biomarkers. American Journal of Obstetrics \& Gynecology. 2018;(2) S725-S732. DOI link: https://doi.org/10.1016/J.AJOG.2017.12.002

4. Figueras F, Caradeux J, Crispi F, Eixarch E, Peguero A, Gratacos E. Diagnóstico y vigilancia de la restricción del crecimiento fetal de inicio tardío. Am J Obstet Gynecol. 2018 ;218(2S):S790-S802.e1. doi: 10.1016/j.ajog.2017.12.003.

5. Debora F.B. Leite, Jose G. Cecatti, "Predicción de restricción del crecimiento fetal: Cómo ir más allá", The Scientific World Journal. 2019;" Artículo ID 1519048, 8 páginas, 2019 https://doi.org/10.1155/2019/1519048

6. Expert Panel on Women's Imaging:, Shipp TD, Zelop CM, Maturen $K E$, Deshmukh SP, Dudiak KM, Henrichsen TL, Oliver ER, Poder L, Sadowski EA, Simpson L, Weber TM, Winter T, Glanc P. ACR Appropriateness Criteria ${ }^{\circledR}$ Growth Disturbances-Risk of Fetal Growth Restriction. J Am Coll Radiol. 2019 ;16(5S):S116-S125. doi: 10.1016/j.jacr.2019.02.009.

7. Dall'Asta, A., Brunelli, V., Prefumo, F. et al. Restricción del crecimiento fetal de inicio temprano. salud materna, neonatol y perinatol. 2017;(2):3. https://doi.org/10.1186/s40748-016-0041-x

8. Scacchi M, Van der Velde J, Vergara R, Rivas M, Sabrina Analís, López Mautino P. Restricción de crecimiento intrauterino. Rev. Hosp. Mat. Inf. Ramón Sardá 2020;1(5).

Microsoft Word - 6 guia.docx (sarda.org.ar)

9. Saldaña Díaz, Jesús lázaro. Factores de riesgo asociados a restricción de crecimiento intrauterino en neonatos atendidos en el servicio de neonatología del Hospital Honorio Delgado, Arequipa, 2017. [Tesis para optar el grado de Doctor]. Arequipa: Universidad Nacional de San Agustín de Arequipa, Escuela de Posgrado;2018.

http://bibliotecas.unsa.edu.pe/bitstream/handle/UNSA/8310/MD Dsadij|2.pdf? sequence $=1$ \&isAllowed $=y$

10. Pimiento Infante Laura Marcela, Beltrán Avendaño Mónica Andrea. Restricción del crecimiento intrauterino: una aproximación al diagnóstico, seguimiento y manejo. Rev. chil. obstet. ginecol. [Internet]. 2015; 80( 6 ): 493-502. Disponible en: https://scielo.conicyt.cl/scielo.php?script=sci_arttext\&pid=S0717$7522620015500006000010 \% 1 \mathrm{ng}=\mathrm{e} s$ http://dx.doi.org/10.4067/S0717-75262015000600010

11. Gomella. Cunninghan. Eyal . Zenk. Neonatology. 5ta Edición

12. Nelson Kliegman. Tratado de Pediatría $20^{\circ}$ edición, 2015

13. Ceriani C. Neonatología práctica 4ta edición, 2009

14. Nelson Kliegman. Tratado de Pediatría $20^{\circ}$ edición, 2015

15. Sola A. Cuidados neonatales 1ra Ed, 2011

16. Martínez-Rodríguez P, Oliva-Cáceres L. Flujometría Doppler en Medicina Fetal. Materno Fetal. REV MED HONDUR.

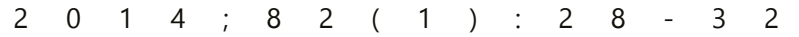
http://www.bvs.hn/RMH/pdf/2014/pdf/Vol82-1-2014-9.pdf

17. Maggio, Lindsay MD; Dahlke, Joshua D. MD; Méndez-Figueroa, Dr Héctor; Albright, Catherine M. MD; Chauhan, Suneet P. MD; Wenstrom, Katharine D. MD Resultados perinatales con relaciones normales en comparación con elevadas de la arteria umbilical sistólica a diastólica en la restricción del crecimiento fetal, Obstetricia y ginecología: 2015;125(4):863-869 doi: 10.1097 / AOG.0000000000000737

18. Martínez P. y Oliva L. Flujometría Doppler en Medicina Materno Fetal. REV MED HONDUR. 2014;82(1):28-32.
19. Simpson L, Khati NJ, Deshmukh SP, Dudiak KM, Harisinghani MG, Henrichsen TL, Meyer BJ, Nyberg DA, Poder L, Shipp TD, Zelop CM, Glanc P. ACR Appropriateness Criteria Assessment of Fetal Well-Being. J Am Coll Radiol. 2016 Dec;13(12 Pt A):1483-1493. doi: 10.1016/j.jacr.2016.08.028. Epub 2016 Oct 28.

20. Ventura Laveríano Walter, Mazarlo Redondo Conny. Significado clínico del Doppler patológico en la arteria cerebral media en fetos del tercer trimestre. Rev. chil. obstet. ginecol. [Internet]. 2010 [citado 2020; 75( 6 ): 405-410. Disponible en: https://scielo.conicyt.cl/scielo.php?script=sci_arttext\&pid=S0717-

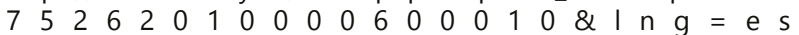
http://dx.doi.org/10.4067/S0717-75262010000600010.

21. Caceres Limachi, Cesar. Validez diagnóstica de la fujometria Doppler como predictor de restricción del crecimiento intrauterino en gestantes con preeclampsia del Hospital Manuel Nuñez Butron de Puno 2020. [Tesis]. Puno: Universidad Nacional del Altiplano, Escuela de Segunda Especialización; 2020 http://repositorio.unap.edu.pe/handle/UNAP/13983

22. Ruoti Cosp Miguel. Evaluación ecográfica del ductus venoso fetal y sus aplicaciones Clínicas en la Obstetricia actual. An. Fac. Cienc. Méd. (Asunción) [Internet]. 2020; 51( 3 ): 99-112.

Available

from: http://scielo.iics.una.py/scielo.php?script=sci_arttext\&pid=S1816-

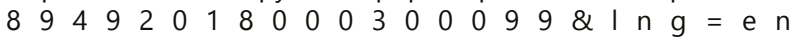
http://dx.doi.org/10.18004/anales/2018.051(03)99-112.

23. Lees CC, Marlow N, van Wassenaer-Leemhuis A, Arabin B, Bilardo CM, Brezinka C, Calvert S, Derks JB, Diemert A, Duvekot JJ, Ferrazzi E, Frusca T, Ganzevoort W, Hecher K, Martinelli P, Ostermayer E, Papageorghiou AT, Schlembach D, Schneider KT, Thilaganathan B, Todros T, Valcamonico A, Visser GH, Wolf H; TRUFFLE study group. 2 year neurodevelopmental and intermediate perinatal outcomes in infants with very preterm fetal growth restriction (TRUFFLE): a randomised trial. Lancet. 2015;385(9983):2162-72. doi: 10.1016/S0140-6736(14)62049-3.

24. Figueras F, Gratacós E. Actualización sobre restricción del crecimiento fetal Protocolo de manejo basado en etapas. Fetal Diagn Ther 2014; 36:86-98 DOI: 10.1159/000357592

25. Acharya G, Tronnes A, Rasanen J. Aortic isthmus and cardiac monitoring of the growth-restricted fetus. Clin Perinatol. 2011:38(1):113-25, vi-vii. doi: 10.1016/j.clp.2010.12.006.

26. Morales-Roselló J, Khalil A. Fetal cerebral redistribution: a marker of compromise regardless of fetal size. Ultrasound Obstet Gynecol 2015; 46:385. https://doi.org/10.1002/uog.15664

27. Regan J, Masters $H$, Warshak CR. Estimation of the growth rate in fetuses with an abnormal cerebroplacental ratio compared to those with suspected growth restriction without evidence of centralization of blood flow. J Ultrasound Med. 2015;34(5):837-42. doi: 10.7863/ultra.34.5.837.

28. Khalil AA, Morales-Rosello J, Elsaddig M, Khan N, Papageorghiou A, Bhide A, Thilaganathan B. The association between fetal Doppler and admission to neonatal unit at term. Am J Obstet Gynecol. 2015;213(1):57.e1-57.e7. doi: 10.1016/j.ajog.2014.10.013. Epub 2014 Oct 15

29. Valenti; Avila; Amenábar, Zanuttini, Crespo. Actualización de Consenso de Obstetricia FASGO 2017 "RCIU (Restricción de crecimiento intrauterino)" Actualizacion_consenso_RCIU_FASGO_2017.pdf

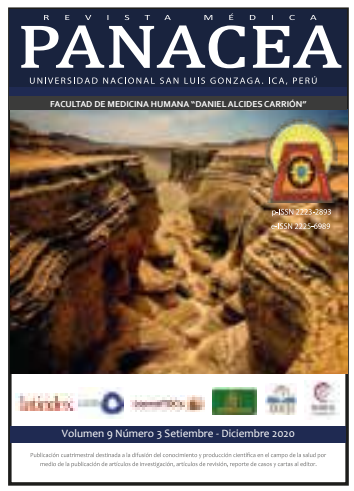

